\title{
2 Human enteric viruses in polluted water
}

Over 120 different viruses are excreted in human feces and urine, find their way into sewage, and become water pollutants. They are listed in Table 1 together with diseases that they cause. The term 'enteric virus' is applied to any viruses disseminated by the fecal route. They are divided into several groups, based on morphological, physical, chemical and antigenic differences.

\section{Enteroviruses}

The most commonly studied group in natural waters has been the picornaviridae family, which includes poliovirus, coxsackie virus $\mathrm{A}$ and $\mathrm{B}$, echovirus, and the high numbered enteroviruses (types 68 to 72 ). These viruses multiply primarily in the alimentary tract and are excreted in substantial amounts (as many as $10^{10}$ per gram of feces) for varying periods of times, with a mean shedding period of up to 50 days. The best studied of these enteroviruses are the polioviruses and the least studied are the many types of group A coxsackie viruses, which are usually isolated in suckling mice. Numerous studies have readily demonstrated the presence of enteroviruses in sewage, in effluents from sewage treatment plants, in contaminated rivers and lakes and in treated drinking waters from developing as well as developed countries (refer to Chapter 3).

Polioviruses can cause serious nervous system disease. Clinical disease occurs only in between 1 in 100 and 1 in over 1000 cases of infection, depending chiefly on the virulence of the virus and the age of the host. At present in countries in which live poliovirus vaccine is widely used, the excreted polioviruses are usually vaccine-derived and their pathogenicity is low, although reversion to varying degrees of neurovirulence during several human passages may occur. The other enteroviruses can also cause nervous system disease, usually of a transient nature (aseptic meningitis) but on occasion clinically similar to typical paralytic poliomyelitis. Some enteroviruses, such as enterovirus type 71, have given rise to large outbreaks of central nervous system disease characterized by encephalitis, or paralysis, or both, with many fatalities. Group B coxsackie viruses cause epidemic pleurodynia (Bornholm disease), pericarditis (chiefly in older persons), serious and often fatal myocarditis in infants and congenital cardiac defects in infants born of mothers experiencing infection during pregnancy. In addition, mild respiratory disease may result from certain enterovirus infections. In summary, enteroviruses can cause serious diseases, but fortunately, under most circumstances, do so in only a very small proportion of infections. A comprehensive review updating our knowledge of enteroviruses and their epidemiology has appeared recently (Melnick, 1985).

This infrequent association with severe disease may well help to explain why reports of the waterborne spread of enteroviruses have been so few. Furthermore, severity of the outcome of infection may be directly related to host 
age. In developing countries, in which wild polioviruses are still prevalent, infections are typically acquired very early in life, when the risk of serious disease is lowest. Most older children and adults are thus immune. In these areas waterborne spread undoubtedly occurs and may be a significant factor in the process of natural immunization. As sanitation has improved in some of these areas, paralytic poliomyelitis has increased, presumably because infections are delayed to an older age. Data on the prevalence of immunity to the enteroviruses other than poliomyelitis are fragmentary, but several studies suggest that urban populations in particular are frequently exposed to these agents.

\section{Hepatitis A virus}

Hepatitis type A virus (HAV) has been proven beyond doubt to be responsible for waterborne epidemics traced to sewage contamination. The virus is a $27 \mathrm{~nm}$ RNA virus whose physico-chemical characteristics place it in the enterovirus group, where it has been classified as type 72 . This virus is excreted in feces over a relatively extended period and has been responsible for waterborne epidemics traced to sewage contamination. The largest waterborne epidemic of hepatitis recorded is the New Delhi outbreak of 1955-56, in which about 30,000 cases were reported, although recent evidence indicates that this outbreak may not have been associated with HAV (Wong et al., 1980). Extensive surveillance in the United States revealed fifteen outbreaks of water associated hepatitis A between 1971 and 1978, which accounted for only $6 \%$ of 224 outbreaks of waterborne disease in the USA during that period. Mosley reviewed some 50 documented water-related epidemics of hepatitis A virus between 1958 and 1964.

Viruses in faecally polluted water accumulate in shellfish in concentrations several times higher than in surrounding waters. Many outbreaks of viral hepatitis have been traced to the ingestion of raw or inadequately cooked shellfish.

During the past four decades unsuccessful attempts to identify and to characterize the hepatitis A virus have frustrated virologists. The reason for this failure was mainly the lack of a laboratory technique for detection and quantification of the agent. Up to the end of the 1960s, when HAV finally could be transmitted to marmoset monkeys, infection of human volunteers was the only means to identify samples containing the virus. Analysis of the physico-chemical properties of the virus became feasible with the detection by immune electron microscopy of virus-like particles with the antigenic properties of HAV in human feces by Feinstone and coworkers in 1973. This key achievement initiated the development of a method for the quantification of hepatitis A antigen by solid-phase radio immunoassay and finally culminated in the cultivation of HAV in vitro in a variety of cell cultures, such as human hepatocellular carcinoma cells, human diploid fibroblast, human amnion, fetal rhesus kidney cells and African green monkey kidney (AGMK) cultures. Virus infection in vitro results in no cytopathic effects but establishes persistent infection. Such persistently infected cultures of AGMK cells are a good source of HAV (Simmonds, 1985). 


\section{Non-A, Non-B hepatitis}

The name 'non-A, non-B hepatitis' includes infections of the liver caused by at least two viruses. These viruses have been characterized as 'blood transmitted', 'coagulation-factor transmitted', and 'epidemic waterborne'. A universally recognized specific serological test has not yet been developed for any of the viruses of non-A, non-B hepatitis. The diagnosis of non-A, non-B hepatitis remains based on the exclusion by serological tests for hepatitis $A$ virus, hepatitis $B$ virus, hepatitis delta virus, cytomegalovirus, and Epstein-Barr virus. A recent review highlights the important aspects of non- $A$, non-B hepatitis virus in relation to the other viruses that may cause hepatitis (Hollinger et al., 1985).

Waterborne non- $A$, non-B hepatitis virus has been the cause of five epidemics in India, an epidemic in Nepal, and sporadic cases in India and the Soviet Union. Its epidemiology resembles that of the hepatitis A virus (HAV), but it is serologically unrelated to it and it can infect patients who have recovered from hepatitis $\mathrm{A}$. Infection has been transmitted by water supplies contaminated by infected feces; cases among household contacts suggest that person-to-person spread may also occur. This virus does not seem to cause chronic infections. The viral etiology was shown and the stool-borne transmission was confirmed by filtered feces obtained from nine patients between the first and the eighth days of jaundice. Immune electron microscopy studies in which convalescent serum from the volunteer was used as a source of antibody demonstrated spherical virus particles with a diameter of 27 to $30 \mathrm{~nm}$ in stools obtained from the volunteer between 28 and 45 days after ingestion. Antibodies were detected in patients with an illness clinically like hepatitis A, in which no IgM anti-HAV was detected. A 27 $\mathrm{nm}$ virus particle in stools and the development of antibodies to it were confirmed in the Nepal epidemic and in the course of infection in a small percentage of marmosets inoculated intravenously with a suspension of stools collected during the epidemic.

The fatality rate may be as high as $12 \%$, although in some outbreaks it may be less than $1 \%$. Epidemic waterborne non-A, non-B hepatitis is most virulent among pregnant women, in whose case fatality rates average $20 \%$ but have reached $39 \%$. Although cases of infection with this virus in western countries have not been reported, there is evidence of an association between shellfish consumption and some cases of non-A, non-B hepatitis in two studies from the USA and the UK. It is possible that these were sporadic cases of epidemic waterborne non-A, non-B hepatitis. Cases of non-A, non-B hepatitis associated with shellfish consumption in western countries have not been analyzed separately to determine whether they are clinically similar to epidemic waterborne non-A, non-B hepatitis.

\section{Norwalk and Norwalk-like viruses}

Since the first identification of the Norwalk virus during an outbreak of gastroenteritis in an elementary school in Norwalk, Ohio, eight new viruses, capable of producing the same type of human viral diarrhea as the Norwalk virus, have been detected in the stools of patients. They include coronaviruses, caliciviruses, astroviruses and parvovirus-like agents. These viruses are 25 to 30 
$\mathrm{nm}$ in diameter, could not be cultivated in cell cultures and did not produce disease when inoculated into laboratory animals, including primates. The development of a radioimmunoassay blocking technique for Norwalk antibody in 1978 by Greenberg and colleagues provided a sensitive and specific test for studying Norwalk virus infections.

Kaplan et al. (1982) reviewed 74 outbreaks of non-bacterial gastroenteritis investigated by the Centers for Disease Control, Atlanta and attributed $42 \%$ of them to Norwalk virus. Of the Norwalk-related outbreaks, water was responsible in 13 and food in 4 . The sources of water included municipal as well as semi-public water supplies and recreational swimming water. In Australia, an outbreak of gastroenteritis from oyster-associated Norwalk virus involved 2000 cases during 1979. Seroepidemiological surveys have shown that acquisition of antibodies to Norwalk virus in developed countries begins slowly in childhood and accelerates during adult life. In contrast, infection with this virus occurs early in life in developing countries.

\section{Rotaviruses}

Rotaviruses have been found to be the major pathogen in non-bacterial infantile diarrhea throughout the world (Flewett and Woode, 1978). At least $50 \%$ of hospitalized cases of gastroenteritis in infants and young children are due to rotavirus infections. Infection has also been reported among adults. Contaminated water has been incriminated in rotavirus diarrhea in the USA, USSR, and Brazil.

The virus particles are approximately $70 \mathrm{~nm}$ in diameter, contain double-stranded RNA and represent a separate genus in the family Reoviridae. The virus particle has a double capsid layer, a smooth rim (outer capsid) surrounding spoke-like subunits (inner capsid) radiating outwards from a hub-like core. Because of the wheel-like appearance, the term rotavirus is used for this virus. Enzyme immunoassay suggests that there are two major antigenic types of rotavirus, whereas by neutralization tests at least three and perhaps four subtypes have been identified. Infection caused by one subtype does not appear to protect against infection by the other.

Highest levels of rotavirus are excreted between the second and fourth day of illness (up to $10^{10}$ particles per gram feces), but a week or so after the onset of symptoms very little virus is detected in stools. The incidence of rotavirus infection in temperate climates peaks during winter months whereas in some tropical developing countries considerable variation in the seasonal distribution of cases has been noticed. Evidence to date indicates that rotavirus infection spreads by the fecal-oral route.

\section{Adenoviruses}

Adenoviruses are also found in the feces and have been isolated from domestic sewage. These are large double-stranded DNA viruses that cause respiratory and eye infections among bathers in nonchlorinated swimming pools. In 1975 , previously unrecognized adenoviruses were detected by electron microscopy in 


\section{Environmental Virology}

stool samples from infants with diarrhea by Flewett and colleagues. These adenovirus species have been designated enteric adenoviruses (EAds). They are fastidious and cannot be cultivated in cell cultures by conventional means. In several studies EAds have been found in 3.9 to $12 \%$ of the stool specimens from infants and young children with acute gastroenteritis. Hospital outbreaks of diarrhea caused by EAds have also been described. Antibodies against EAds have recently been shown to be common and widespread throughout the world.

Recently, EAds have been propagated in selected cell cultures, namely 293 cells, Chang conjunctival cells, and African green monkey kidney cells. Two distinct types of EAds, AD40 and AD41, have now been identified by ELISA and electron microscopy. Uhnoo and coworkers (1984) conducted a one-year study of acute infantile gastroenteritis in children up to fifteen years of age at the University Hospital at Uppsala, Sweden, and detected adenoviruses in the stools or by seroconversion, or both, in 56 of $416(13.5 \%)$ of ill children. Of the adenoviruses detected, $59 \%$ of the specimens were identified as adenoviruses 40 and 41. Evidence for established adenoviruses (Ad1 through Ad39, in gastroenteritis was found in 15 of $416(3.6 \%)$ ill children. The predominant symptom of infections with enteric adenoviruses was diarrhea, with a mean duration of 8.5 days (Ad40) and 12.2 days (Ad41). One-third of the children with AD41 infections had prolonged symptoms ( $>14$ days). The established adenoviruses ( 1 to 39) presented a different clinical picture, characterized by diarrhea of shorter duration, higher fever, and significantly increased occurrence of respiratory symptoms $(79 \%)$. In this study, adenoviruses were observed throughout the year and displayed no marked seasonal pattern, in contrast to the winter prevalence of rotaviruses. AD40 and AD41 showed similar seasonal distribution with a small peak in July (warmer month), where one-third of all gastroenteritis was due to EAds. The role of EAds in infantile gastroenteritis in developing countries is not known at this time.

\section{Parvoviruses}

The adeno-associated viruses (AAV) were the first parvoviruses of human origin to be discovered. AAV, together with adenoviruses, have been recovered from feces, and hence may be present in polluted water. However, data on the frequency of excretion of these viruses are lacking. Seroepidemiological studies indicate that antibodies to AAV, especially types 2 and 3 , are widely prevalent in young children.

\section{Enteric viruses in the etiology of waterborne disease}

Acute gastrointestinal and diarrheal illnesses continue to be the major waterborne diseases in the more developed countries, usually with no etiological agent being identified. Recently, seroepidemiological studies have identified Norwalk virus as the probable cause in several waterborne oubreaks of gastroenteritis. Rotaviruses have also been suspected. Hepatitis A virus (enterovirus type 72 ) is the only other viral agent clearly recognized as having caused waterborne disease outbreaks. Several very large outbreaks of drinking-watertransmitted hepatitis have been recognized in India. Outbreaks of swimming pool 
adenovirus pharyngoconjunctivitis also have been documented, in which improperly chlorinated and maintained swimming pools were involved. While waterborne transmission of poliovirus and other enteroviruses has been suspected, conclusive evidence has been lacking.

Waterborne outbreaks due to enteroviruses and other enteric viruses are not easily recognized and are difficult to document for several reasons: (i) Many of these viruses cause inapparent infections that are difficult to recognize as being waterborne. A person may contract a viral infection by ingesting contaminated water, and the virus may actively multiply in the alimentary tract without the person developing overt symptoms of the disease. The person may have only mild malaise for a few days or no symptoms at all, yet may act as an effective carrier and transmit the virus to others, who may then develop acute symptoms of the disease. (ii) Epidemiological techniques are not sufficiently sensitive to detect low-level transmission of virus diseases through water. As shown in Table 1, most enteric viruses cause such a broad spectrum of disease syndromes that scattered cases of acute illness would probably have symptoms too varied to be attributed to a single etiological agent.

Table 1 Human viruses that may be present in polluted water

\begin{tabular}{ll}
\hline Virus group & $\begin{array}{l}\text { Number of Disease caused } \\
\text { serotypes }\end{array}$ \\
\hline
\end{tabular}

\section{Enteroviruses:}

Poliovirus

Echovirus

Coxsackievirus A

Coxsackievirus B

New enterovirus types 68 to 71

Hepatitis A (enterovirus 72)

Norwalk virus

Rotavirus

Reovirus

Parvovirus:

adeno-associated virus

Adenovirus (feces and urine)

Cytomegalovirus (urine only)

Papovavirus, SV40-like (urine only)
Paralysis, meningitis, fever Meningitis, respiratory disease, rash, fever, gastroenteritis Herpangina, respiratory disease, meningitis, fever, hand, foot and mouth disease Myocarditis, congenital heart anomalies, rash, fever, meningitis, respiratory disease, pleurodynia Meningitis, encephalitis, respiratory disease, rash, acute hemorrhagic conjunctivitis, fever Infectious hepatitis Epidemic vomiting and diarrhea, fever Gastroenteritis, diarrhea Unknown

\section{Unknown}

Respiratory disease, conjunctivitis, gastroenteritis

1 Infectious mononucleosis, hepatitis, pneumonitis, immunological deficiency syndrome Associated with progressive multifocal leukoencephalopathy and immunosuppression 


\section{Environmental Virology}

Because of the inadequacy of surveillance systems and methods, only a small percentage of enteric viral infections would be expected to be reported or investigated. The importance of low-level transmission is difficult to document because of the occurrence of large numbers of asymptomatic or subclinical cases characteristic of the enteric viruses. However, its importance in maintaining a disease in a community should not be overlooked.

Low level transmission of agents of viral gastroenteritis by water or food may play its most significant role in the spread of these agents between communities distant from each other. For example, viruses may be discharged by one community into a river which is used farther downstream as a water source by another community. Only a few individuals in the downstream community need contract the disease by bathing or water consumption to establish new foci of infection from which viruses can be spread by other routes (i.e. respiratory or fecal-oral). In most cases it is very difficult to identify the point source of viruses that are detected at any distance downstream from their discharge, but sometimes it has been done and the results show that viruses can travel miles from the source of contamination and still be infectious.

\section{Summary}

Over 120 different viruses are excreted in human feces and urine, find their way into sewage, and become common water pollutants. A wide spectrum of diseases including paralysis, meningitis, respiratory disease, epidemic vomiting and diarrhea, myocarditis, congenital heart anomalies, infectious hepatitis and eye infections can be caused by these viruses. Although poliovirus, coxsackievirus $A$ and $\mathrm{B}$, and echoviruses have been the most studied of the enteric viruses, they have not been shown to be involved in waterborne outbreaks.

Hepatitis A virus, rotavirus and Norwalk-like agents have been implicated in several waterborne disease outbreaks. Two types of non-A and non-B hepatitis viruses have been the cause of waterborne hepatitis. Two distinct types of enteric adenoviruses, $\mathrm{AD} 40$ and $\mathrm{AD} 41$, have been recognized as a cause of acute gastroenteritis in children.

\section{References}

Flewett, T. H. and Woode, G. N. (1978). The rotaviruses. Archives of virology 57: 1-3. Hollinger, F. B., Melnick, J. L. and Robinson, W. S. (1985). Viral Hepatitis: Biological and Clinical Features, Specific Diagnosis and Prophylaxis. Raven Press, New York.

Kaplan, J. E., Gary, G. W., Baron, R. C., Singh, N., Schonberger, L. B., Feldman, R. and GREENBERG, H. B. (1982). Epidemiology of Norwalk gastroenteritis and the role of Norwalk virus in outbreaks of acute nonbacterial gastroenteritis. Annals of Internal Medicine 96: 756-61.

MelNiCK, J. L. (1985). Enteroviruses: polioviruses, coxsackieviruses, echoviruses, and newer enteroviruses. In: Virology (edited by B. N. Fields, D. M. Knipe, R. M. Chanock, J. L. Melnick, B. Roizman and R. E. Shope). Raven Press, New York, pp. 739-94.

Simmonds, R. S., Szucs, G., Metcalf, T. G. and Melnick, J. L. (1985). Persistently infected cultures as a source of hepatitis A virus. Applied and Environmental Microbiology 49: 749-55. 
Uhnoo, I., Waddel, G., Svenson, L. and Johansson, M. E. (1984). Importance of enteric Adenoviruses 40 and 41 in acute gastroenteritis in infants and young children. Journal of Clinical Microbiology 20: 365-72.

Wong, D. C., Purcell, R. H., Sreenivasan, M. A., Prasad, S. R. and Pavri, K. M. (1980). Epidemic and endemic hepatitis in India: evidence for a non-A, non-B hepatitis virus etiology. Lancet 2: 876-9. 